\title{
STOCHASTIC TRAJECTORIES OF SPACE VeHICLES WITH GRAVITATIONAL MANOEUVRES
}

\author{
A.V. Krivov, L.I. Sokolov, and V.B. Titov \\ Astronomical Observatory, St. Petersburg State University
}

\begin{abstract}
The theoretical a spect of close flybies of space-crafts near planets-gravitational ma noeuvres have been studied. It is proved that the space-craft motion with gravitational manouvres admits the existence of routing scheme and, consequently shows the existence of quasi-random motion.
\end{abstract}

\section{INTRODUCT ION}

The practical application of close flybies of spacecrafts near planets - gravitational manoeuvres - is wide-spread and u sual in space research. Gravitational ma noeuvres minimize flytime or fuel expense and give diverse opportunities to $\mathrm{ch}$ oose variants of space missions.

This is the appl ied a spect of the problem. But it is not less interesting from theoretical view point: the trajectories with numerous flybies prove to be an example of stochastic motions in classical deterministic dynamical system. It is this side of the problem that is under consideration in the present paper.

The cause of stochasticity is, of principle, evident: this is instability, leading to the expansion of initial tube of trajectories in the course of time (Fig. 1). The negligible small deviations of elements of initial or bit result in small changes of impact parameter, but the latter cause considerable changes of or bital elements after encounter. As a result, the dynamical system rapidly "forgets" its initial state. 


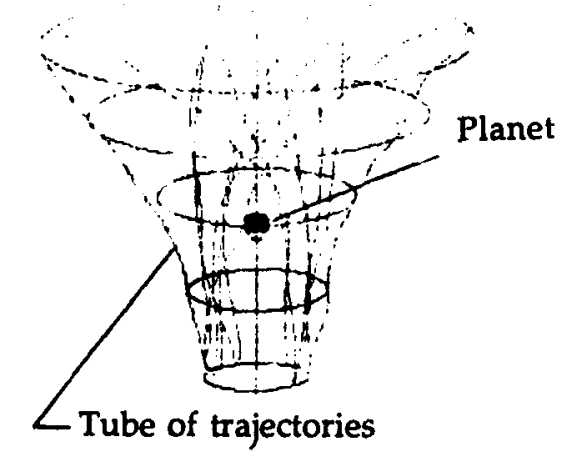

Fig. 1. The cause of stochasticity is unstability leading to the expansion of initial tube of trajectories.

Let $u s$ start with giving more rigorous mathematical meaning to the statement "the motion has stochastic properties". Introduce the concept "qua si-random motion", following V.M. Alekseev (1981). Suppose the dynamical system allows the transformation to a system with discrete time $t \varepsilon\left\{t_{n}\right\}_{n=-\infty}^{\infty} i . e$. to the iterations of some phase space transformation. Let us assume, further, that any motion is such as $\forall n x_{n} \equiv x\left(t_{n}\right) \varepsilon \ell_{n^{\prime}}$ $L \equiv u l_{i}$ being the totality of non-overlapping subsets of the pha se space. Hence, any motion is to be represented by a sequence of the "letter $s$ " of the "alphabet" $L$ :

$$
\ldots \ell_{1} \ell_{2} \ell_{3} \ldots
$$

The sequence for which corresponding motion exists is called admissible. A part of admissible sequence of the length 2 (i.e.consisted of two "letters") is called admissible transition. One can say that the motion is quasi-random if it's impossible to predict the next letter of the sequence, pro$v i d e d$ the fragment of admissible sequence of arbitrary length is known.

The another (probably,more understandable) way to describe these ideas is to use the notion of routing scheme. The latter is the oriented graph, its modes being the "letters" of the "alphabet" L, and the presence of arc (with arrow prescribed) between two nodes means that corresponding transition is admissikle. The motion is called quasi-random if it may be represented by routing scheme with two or more arrows starting from each node (Fig.2). 


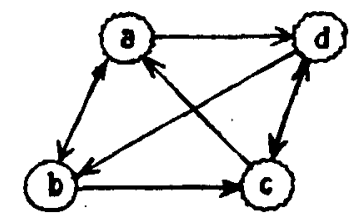

Fig. 2. An example of quasi-random motion represented by routing scheme.

If the part of the admissible sequence is known (e.g., ...abc...), it is impossible to find its unique continuation. The continuation ...abca... is admissible as well as ...abcd....

our goal is to prove that the problem of spacecraft motion with gravitational manoeuvres admits the existence of routing scheme and,consequently, there exist quasi-random motions in this problem.

\section{THE MODE}

Let us consider the simplest problem of celestial mechanics revealing stochastic properties - the plane circular restricted three-body problem Sun-planet-spacecraft (Fig. 3). Moreover, for the sake of simplicity let us treat now the

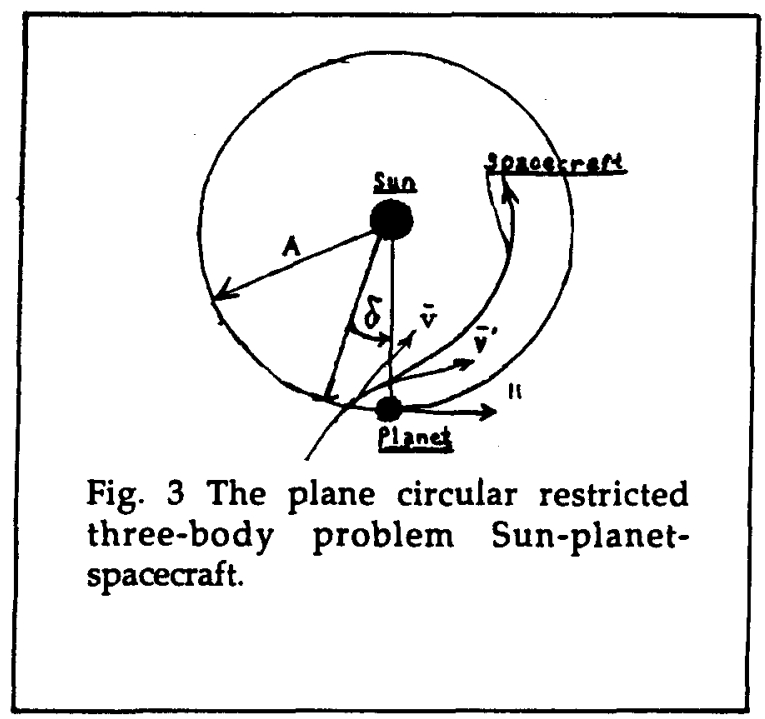


approximate model of this protlem lalthough the latter is already a model problem). Namely, we shall u se the method of action spheres with zero radii lor, briefly, point-like action spheres method). It can be summarized as follows. The spacecraft motion is supposed to be Keplerian hel iocentric everywhere except for po ints of encounter with planet, where instant change of velocity vector occurs. This change is reduced to the rotation of velocity vector to the angle $\psi$ (between the a symtotes of pla netocentric hyperbola). Any flyby, therefore, looks like a "collision" of spacecraft with planet.

Let heliocentric gravitational constant be equal to unity whereas planetocentric one equals to $\mu$. The planet moves on the circular or bit of radius $A$ with linear velocity $u=1 /$ $\checkmark \bar{A}$. Denote heliocentr ic velocity vector of spacecraft as $\bar{v}$, planetoc entric one as $\bar{w}$.

It is easy to deduce formulas expressing the change of spacecraft or bit as a result of a collision with planet:

$$
\begin{aligned}
& v^{\prime 2}=v^{2}+\frac{4 u z}{1+z^{2}}\left[z\left(u-v^{(n)}\right)-v^{(x)}\right] \\
& v^{(n)}=\left(v^{\prime 2}+u^{2}-w^{2}\right) / 2 u \\
& \left.\left.v^{(x)}\right)^{2}=v^{\prime 2}-v^{(n)}\right)^{2}
\end{aligned}
$$

here the prime indicates to values immediately after collision, whereas its a bsence corresponds to values directly before it. The quantity $z \equiv \tan (\psi / 2)$ may be referred to as flyby parameter. It is connected with the impact parameter $\rho$ by known relation

$$
z=\mu / w^{2} \rho
$$

One can obtain an approximate expression for $p$ by means of I inearization of spacecraft motion in the vicinity of the planet. With technical details omitted, the final result is

$$
\rho \approx A|\delta|\left|v^{(r)}\right| w^{-1}
$$

$\delta$ being the angular distance from spacecraft to planet while the unperturbed spacecraft is crossing the planet or bit.

Let us recall, finally, the well-known Keplerian relations:

$$
\begin{aligned}
& v^{2}=2 / A-1 / a, \\
& v^{(n)^{2}}=a\left(1-e^{2}\right) / A^{2},
\end{aligned}
$$


with a and e standing for major semiaxis and eccentricity of spacecraft or bit, respectivel y.

The set of formulas (1)-(7) completely describes the transformation of Keplerian heliocentric or bit of spacecraft as a result of a flyby:

$$
(a, e) \rightarrow\left(a^{\prime}, e^{\prime}\right)
$$

\section{STOCHAST IC PROPERTIES}

In many well-known stochastic dynamical system s the repetition of collisions is guaranteed automatically (e.g. in billiards). It is not the case here: we ought to take care of such a repetition. Namely, one have to $c$ boose the parameters of each flyby such as to reach next flyby in the nearest future.

This condition is satisfied particularly for resonant orbits, for which the next collision occurs at the same point a s previous one after $\vec{p}$ completesrevolutions of planet and $q$ completes revolutions of spacecraft:

$$
(\mathrm{a} / \mathrm{A})^{3 / 2}=\mathrm{p} / \mathrm{q}, \mathrm{p}, \mathrm{q} \in \mathbb{N}
$$

The non-resonant orbits (al so containing the orbits of collision) vere also investigated (Sokolov, 1990) but are not discu ssed here.

Restricting ourselves by the consideration of resonant or bits we have:

$$
\begin{aligned}
& \delta_{i+1}=\delta_{i}+2 \pi A^{-3 / 2}\left[q_{i}\left(2 / A-v_{i}^{2}\right)^{-3 / 2}-p_{i} A^{3 / 2}\right] \\
& z_{i+1}=\frac{\mu}{A\left|\delta_{i+1}\right| v_{i}^{(r)}\left(v_{i}^{2}+u^{2}-2 u v_{i}^{(n)}\right)^{1 / 2}} \\
& v_{i+1}^{2}=v_{i}^{2}+\frac{4 u z_{i+1}}{1+z_{i+1}^{2}}\left[z_{i+1}\left(u-v_{i}^{(n)}\right)-v_{i}^{(n)}\right] \\
& v_{i}^{(n)}=\left(v_{i}^{2}+u^{2}-w^{2}\right) / 2 u, v_{i}^{(r)^{2}}=v_{i}^{2}-v_{i}^{(n)^{2}}
\end{aligned}
$$

Here $i=1,2,3, \ldots$ stands for the mumber of collision, $v_{i}=\left\{v_{i}^{(n)}, v_{i}^{(r)}\right\}$ is the velocity after $i-t h$ collision (the same as the velocity directly before $i+1-t h$ collision, because the orbits are resonant). 
It can be easily shown that for each set of pairs $\left(p_{j}, q_{j}\right)$, $i=1,2,3, \ldots$, the system of equations $(9)-(12)$ has an exact solution, provided $\mu$ is sufficiently small. In order to show it, let us $f$ ix the value of planetocentric velocity $w$ ( $w$ is an invariant, because its values before and after each collision coincide). Choose an arbitrary sequence of resonant heliocentric velocity values $v_{i}$ (corresponding to the values of $p_{i}, q_{i}$ chosen from the interval $(u-w, u+w)$, restricting our selves by not too large values of $p_{i}, q_{i}$. Evaluate normal and radial components $v_{i}^{(n)}$ and $v_{i}^{(r)}$ by means of $(12)$. The quadratic equation $(11)$ determines $z_{i}$ lone can prove the existence of real roots). The next step is to calculate $\delta_{i}$ with the aid of (10). Finally, we use formula (9) either $i$ to obta in $\delta_{1}$ (when $i=1$ ) or to correct the value of $v_{i}$ (when $i=2,3, \ldots)$. The corrections to $v_{i}^{(n)}$ and $v_{i}^{(r)}$ are to be found now from (12). These operations are to be repeated until the required accuracy is achieved (Fig. 4). This iterative procedure, performed for all transitions, results in the set of values $v_{i}$. Note that the iterations converge as geometric progression with factor $\psi$, independently of the path in the routing scheme. It can be clarified by the presence of small parameter $\mu$ in ( 1.0$)$ which provides the "compression". Indeed, if the correction to $z$ is of order $\mu \mathrm{k}$, the correction to $\delta$ (and $v$ ) is of order $\mu^{k+1}$.

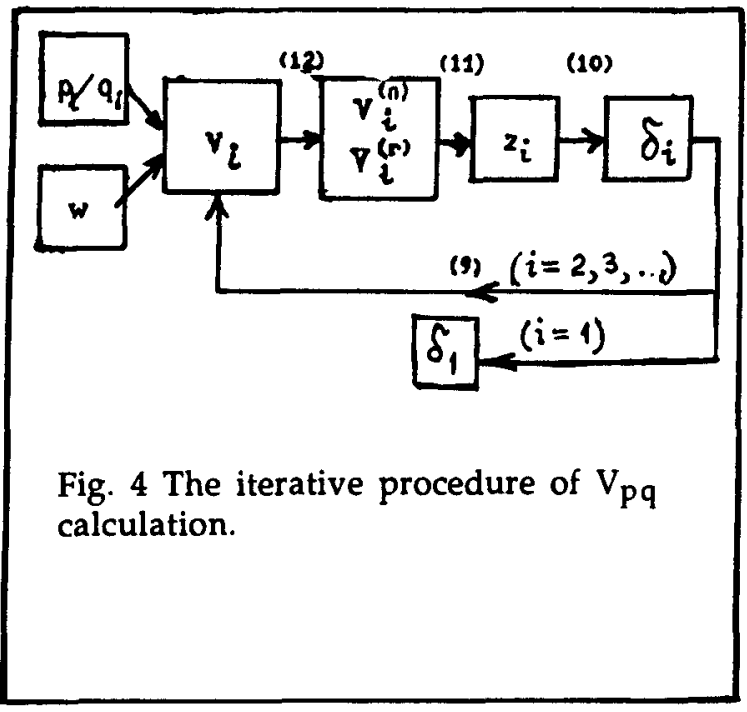


Therefore, the existence of the trajectory corresponding to each sequence of $\left(p_{i}, q_{i}\right)$ is proved. Thus, there exists routing scheme - the infinite oriented graph - with at least two arcs originating in each node (Fig. 5). Te can conclude therefore that the motion is quasi-random according to our definition. All routes are permitted all pairs of nodes are 1 inked by arrows. For each route within this screme there exists a corresponding trajectory in the simplified threebody problem, e.g., the path $1 / 2+2 / 3+1 / 1+\ldots$ on the $F$ ig. 5 may have an arbitrary contimuation. The spacecraft rambles through the nodes of $x$ heme.

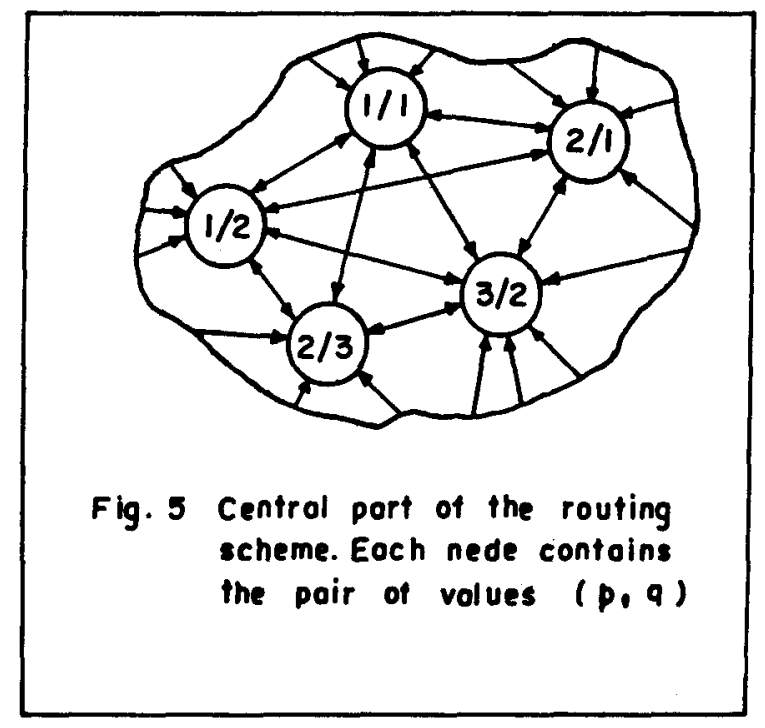

\section{DISCUSSION}

Thus, in the given specific problem of celestial mechanics an example of quasi-random motion has been constructed. It should be noted that we have considered not the "true" (actual) three-body problem but "di storted", "spoiled" by the using of two operations - the point-like action spheres method and 1 inearization of spacecraft motion while evaluating the impact parameter. So the motions under consideration do not exist in reality. Neverthel ess, they approximate actual solutions with sufficient accuracy - the fact that has been proved by the comparison with exact a nalytical results, in 
particular, with Tisserand criterion. It is especially important that quasi-random nature of the motions "survives" in the actual tiree-body problem. Let us quote vladimir Arnold: "Unlike the stability, the instability is stable".

We shall come now to the other consequences of our treatment. It is clear that the mea sure phase space subset where the stochasticity is actually revealed is very mall. On the contrary, the velocity of accuracy loss cau sed by consequent spaceraft flybies is considerable, amounting to $3-4$ decimal orders per one flyby. As a consequence, it is a bsolutely useless to analyze an individual stochastic trajectory by numerical integration if the time interval is not small.

Further, it seems to be evident that the technique u sed in the paper may be generalized to more complicated cases of $\mathrm{N}$-body problem. For example, one can consider four-body problem in which two planets like as Mercury and venus are involved. It doesn't make difficulties al so to study 3 -dimensional (non-planar) problem. The 1 ist of possible generalizations is okviously to be contimued.

The applied a spect of the problem mentioned above is al so of great interest. It includes the investigation of the $r$ egions of attainability, the analysis of the possibilities to fall down to the sun, or to excape from the solar system, or to leave the ecliptic plane.

All these generalizations and a spects of the problem are considered in detail in a series of papers (Sokolov,1980; sokolov, Titov, 1990). These paper s conta in al so the description of the interactive computer system constructing stochastic trajectories. It makes it possible to "grow" trees of trajectories by contiming appropriate branches and stopping bad ones, due to accepted criteria. Numerical examples of trajectories constructed using the described system are al so presented.

\section{REFERENCES}

[1] V.M. Alekseev, (1981), Final motions in three body proklem and symbolic dynamics. Uspekhi matematicheskin nauk. v.36, No. 4, p.161-176 (in ku ssian).

[2] L.J.. Sokolov, (1990), The solutions of three-body problem and random processes. Vestnik of Leningrad University "Math. Mech. Astron.", (in press).

[3] L.L.Sokolov, V.B:Titov, (1 990), Spacecraft trajectories with gravitational manoeuvres, Vestnik of Leningrad University Math. Mech. Astron", (in press). 